\title{
PROKLA-Redaktion
}

\section{Editorial: Klassentheorien}

\section{Renaissance der Klassentheorie?}

Bereits vor 20 Jahren vermutete unser geschäftsführender Redakteur Michael Heinrich (1994), wahrscheinlich würden sich nur noch ältere Leser und Leserinnen daran erinnern, dass das Akronym im Titel der Zeitschrift einst für „Probleme des Klassenkampfs" stand. Er fuhr fort, dass sich mit der Änderung des Titels im Jahr 1976 nicht nur der Name des Projekts PROKLA gewandelt habe, sondern auch auf die veränderten gesellschaftlichen Umstände reagiert wurde. Emanzipationsbestrebungen könnten "nicht mehr ausschließlich als 'Klassenkampf' und 'sozialistische Politik' aufgefasst werden", dazu quer liegende Unterdrückungsformen wie Sexismus oder Rassismus, müssten ebenbürtig berücksichtigt werden, um einen „Beitrag zur Veränderung der Verhältnisse [auf der Höhe der Zeit] zu leisten" (ebd.: 110).

Diesem Resümee voraus ging ein selbstkritisches Editorial der Redaktion im Jahr 1985, als die bis dahin letzte Ausgabe mit explizitem Klassenschwerpunkt erschien. Darin war nicht nur von einem „augenscheinlichen Prognosedefizit der marxistischen Klassenbewusstseinstheorie" (PROKLA 1985: 2) die Rede, sondern auch von einer notwendigen „kategorialen Erweiterung der Klassentheorie“ (ebd.: 3), um angemessen berücksichtigen zu können, dass Formationen und Identitäten bestehen, die ökonomische Klassenlagen durchkreuzten und politische Präferenzen nicht einfach aus Klassenpositionen abgeleitet werden dürften.

Wiederum gut 30 Jahre später muss an dieser Stelle betont werden, dass die PROKLA - ohne die erheblichen konzeptionellen Schwierigkeiten in Abrede zu stellen - stets eine Erweiterung oder Fortentwicklung, nicht aber eine Abschaffung der Klassentheorie verfolgte. Klassentheorie blieb ein Teil vieler Artikel der Zeitschrift, was in der hiesigen Debatte keine Selbstverständlichkeit darstellte. Im Rückblick war in der soziologischen Disziplin der Sozialstrukturanalyse ein ideologisch geprägter „westdeutscher Sonderweg“" (Geißler 1992: 324) auffallend, an dessen Ende „vertikale Strukturen wegdifferenziert, wegpluralisiert, wegindividualisiert und wegdynamisiert" (ebd.: 323) wurden. In Bezug auf die Klassentheorie wurde vielfach sprichwörtlich das Kind mit dem Bade ausgeschüttet.

Freilich hat sich dieses Bild in jüngerer Vergangenheit gewandelt. Zwar ist die Klassentheorie auch heute ,in keinem guten Zustand" (Dörre 2010: 198), da die - von Ausnahmen abgesehen (z.B. Giddens 1984; Koch 1994; Kohlmorgen 2004) - lang anhaltende Vernachlässigung ihrer konzeptionellen Ausarbeitung und empirischen Anwendung nicht spurlos an ihr vorübergegangen ist. Doch gibt es so etwas wie ein Comeback der klassentheoretischen Begriffe und Heuristik. Nicht nur ist es im öffentlichen und wissenschaftlichen 
Diskurs wieder gängiger, von Klassen als soziale Gruppen zu sprechen. Gerade die Beschreibung gegenwärtiger Gesellschaften als Klassengesellschaften trifft nach Ansicht vieler - und keineswegs nur der üblichen Verdächtigen - ins Schwarze. So spricht Hans-Ulrich Wehler in der Zeit offen von Vermögensverhältnissen als neuen „Klassengrenzen“ in Deutschland (07.02.2013). In der Tagespresse, z.B. der Frankfurter Allgemeinen Zeitung, ist sogar von einer "neuen Klassengesellschaft" die Rede (Bollmann/Kloepfer 02.06.2013). Selbst bekannte Vertreter und Vertreterinnen der Individualisierungsthese wie Ulrich Beck und Angelika Poferl (Hg., 2010) diskutieren in ihren jüngeren $\mathrm{Pu}$ blikationen Klassentheorien wie die von Immanuel Wallerstein oder Leslie Sklair. Die diskursiven Angriffe auf den Klassenbegriff scheinen also abzunehmen. Die Praxis sieht in Zeiten von Bailouts für die Banken und Austeritätsmaßnahmen für die Armen jedoch anders aus, wie es auch der Großinvestor Warren Buffett offen aussprach: „Es herrscht Klassenkampf, richtig, aber es ist meine Klasse, die Klasse der Reichen, die den Kampf führt, und wir gewinnen" (zit. nach NYT 26.11.2006).

\section{Essentially contested: Der Klassenbegriff}

Die Renaissance des Klassenbegriffs sollte aber nicht darüber hinweg täuschen, dass mehr denn je umstritten ist, was mit „Klasse“ eigentlich bezeichnet werden soll; ferner, wie eine umfassende Klassenanalyse der Gegenwartsgesellschaften aussehen kann. Dies liegt jedoch nicht (nur) an unzureichenden Definitionsversuchen. Wie die Begriffe Kultur, Macht und Politik ist der Begriff der sozialen Klasse ein „essentially contested concept" (Gallie 1956). Denn es ist keine unzweideutige Definition des damit
Gemeinten möglich, der Bedeutungsinhalt ist umkämpft. Dabei handelt es sich nicht um eine Nachlässigkeit im alltäglichen oder wissenschaftlichen Sprachgebrauch. Die Umkämpftheit wird auch nicht im Fortgang der empirischen Forschung und logischen Deduktionen einem konsensualen, neutralen und harmlosen Begriff weichen, obwohl es diesen bloß nominalistischen Klassenbegriff auch gibt. Nein, wer in Begriffen von Klasse redet, trifft damit auch eine bestimmte Entscheidung wie er/ sie den Begriff verstanden wissen möchte. Je nach Erwartung und Vorbegriffen der Beobachterin - Autonomie, Bewusstsein, Subjekt, Struktur, die Bedeutung von Arbeit für das Leben, die Gestalt und Mitgestaltung der konkreten Arbeitsvollzüge etc. - ist jeweils etwas anderes gemeint.

Gilt das grundsätzlich für die Rede von sozialen Klassen, so ist doch auch eine Dynamik im Gebrauch des Begriffs im Rahmen kritischer Gesellschaftstheorie festzustellen. Denn wie alle Kritik hat auch die der Klassengesellschaft in den letzten Jahrzehnten ihre Selbstsicherheit verloren. Wie Klasse erfahren wird, hat sich gewandelt, und auch die Form der Klassenkämpfe ist eine andere. Zudem muss der Begriff heute viel reflexiver in Hinblick auf ungewollte Homogenisierung oder performative Machtwirkungen verwendet werden. Alles das sind Gründe für die theoretische Reflexion. Dabei sehen sich die Kritiker_innen mit der Schwierigkeit konfrontiert, die Macht von Strukturen zu betonen, die im Handgemenge sozialer Kämpfe möglicherweise nicht immer mitgedacht werden. Mit anderen Worten: Der Klassenbegriff stellt auch die wichtige Frage nach dem Verhältnis zwischen Objekt und Subjekt der Kritik, also inwiefern die Kritik mit der Gesellschaft, die sie als Klassengesellschaft kritisiert, und den Menschen, die diese Gesellschaft reproduzieren, verbunden oder eben nicht verbunden ist. 
Trotz aller Interpretationsbedürftigkeit hat der Klassenbegriff einen Bedeutungskern, der Raum für sich wiederholende Debatten mit einer begrenzten Bandbreite von Auslegungen gibt. Dieser Kern besteht in drei Eigenheiten, die den Klassenbegriff gerade auch heute gegenüber konkurrierenden Konzepten der Sozialstrukturanalyse, insbesondere Schicht und Milieu, profilieren: 1) Ausbeutung, 2) Herrschaft und 3) Klassenkampf. Sie haben gemeinsam, dass das Denken in Klassen relational und prozessual ist, nicht die vorliegende Ungleichheit wird quantifiziert, sondern die Mechanismen aufgezeigt, die im Ergebnis zu diesen Ungleichheiten führen.

So verweist der erste Punkt, Ausbeutung, auf den basalen Mechanismus, dass die Einen gerade deshalb mehr haben, weil die Anderen weniger haben. In der kapitalistischen Produktion eignet sich die Klasse der Kapitalist_innen die Mehrarbeit der Lohnabhängigen an und erwirtschaftet so ihren Reichtum - so lautet Marx' einfache Formel, die heute wieder durch Thomas Pikettys umfangreiches Datenwerk in seinem Buch Capital in the Twenty-First Century ungeahnte Popularität gewonnen hat (Piketty 2014). Ferner besteht eine „wechselseitige Abhängigkeit" (Demirović 2008: 54) zwischen den Klassen. Unter kapitalistischen Rahmenbedingungen brauchen sie sich einander, um zu wirtschaften und zu (über-)leben: Finden Kapitalist_innen keine willigen Arbeitskräfte, droht ihnen der Bankrott, finden die Lohnabhängigen keinen Arbeitsplatz, droht ihnen die Erwerbslosigkeit.

Zweitens ist dieses Verhältnis aber kein gleiches, sondern ein asymmetrisches und antagonistisches. Die Klassenanalyse ist immer auch Herrschaftsanalyse, der Klassenbegriff impliziert mehr als eine bloße Schichtung der Gesellschaft. Klassenherrschaft ist vielmehr auf verschiedenen Ebenen präsent, etwa im Direktionsrecht der
„Arbeitgeber_innen“ gegenüber den „Arbeitnehmer_innen“ im Betrieb oder in der politischen Herrschaft. Sie äußert sich im spannungsgeladenen Selektivitätsdruck des bürgerlichen Staats, „der seinen Klassencharakter zugleich praktizieren und unsichtbar machen muss" (Offe 1972: 92). Klassen und Klassenfraktionen ringen in der politischen Sphäre um Hegemonie, die bürgerliche Herrschaft steht stets unter Legitimationsdruck, da die strukturelle Umverteilung von unten nach oben nicht immer von den Beherrschten sprachlos hingenommen wird.

Folglich ist das Klassenverhältnis grundsätzlich spannungsgeladen. Anders als Methoden, die primär mit statistischen Querschnitten arbeiten, geht es in Klassenanalysen deshalb auch um die gesellschaftliche Dynamik, die sich unter anderem in und vermittelt durch Klassenkämpfe vollzieht. Nicht zufälligkommt Marx erst am Ende des dritten Bandes des Kapitals systematisch auf die Klassen zu sprechen. Ihre Konstitution und Ausprägung, letztlich: ihre Bewegung als Klassenkampf ist aus der formanalytischen Methode nicht einfach ableitbar, sondern sie muss als der „vermittelste und daher konkreteste Begriff“" (Demirović 2008: 53) durch die Analyse der verschiedenen Elementarformen des Kapitalismus hindurch gewonnen und historisch-konkret analysiert werden. Marx' emphatische Hoffnung, dass die Bourgeoisie mit dem Proletariat „ihren eigenen Totengräber produziert" (MEW 4: 474) rührte also aus der Konfliktdynamik, die der bürgerlich-kapitalistischen Vergesellschaftung innewohnt.

\section{Ausdifferenzierung in verschiedene Klassenbegriffe}

Ungeachtet des gemeinsamen Bedeutungskerns der Klassentheorie haben sich äußerst heterogene Klassenbegriffe herausgebildet, die nicht ohne weiteres kompatibel sind. 
Dabei sollte die Geschichte der Klassenbegriffe nicht als reine Ideengeschichte missverstanden werden. Denn die historische Transformation der Sozialstruktur und die gesellschaftlichen (Deutungs-)Kämpfe beeinflussen immer auch die Theorieproduktion. So erschwerten in den vergangenen Jahren ein gesellschaftliches Klima und eine Politik, die Individualisierung und Aktivierung diskursiv vorantrieben, die Bedingungen dafür, ebenso wie Prekarisierungsprozesse die Formierung und Thematisierung von Klassen verhinderten. Auch bedingt die Umkämpftheit des Klassenbegriffs, dass in unterschiedlichen Verwendungsweisen unterschiedliche Erkenntnisinteressen zum Ausdruck kommen und Klassifikationskämpfe stattfinden. Zudem unterscheiden sich die Begriffe danach, wie die einzelnen Merkmale gewichtet werden und ob sie bei der Beschreibung der Klassen eher auf die strukturelle Seite (z.B. Besitz an Produktionsmitteln, Stellung im Produktionsprozess etc.), die lebensweltliche Praxis (z.B. milieuspezifische Distinktion etc.) oder die (Nicht-)Bewegung als Kollektivsubjekt (z.B. Gewerkschaftsbewegung etc.) fokussieren.

Die unterschiedlichen Lesarten sind bereits in Marx' Werk angelegt. Nachdem Marx in den Analysen der französischen Klassenverhältnisse um die Zeit der 1848er Revolution eine konkrete Analyse des sozialen und politischen Alltags vorgelegt hatte, ging er zu den Gesellschaftsstrukturen zurück und begann eine umfangreiche Untersuchung der kapitalistischen Produktionsweise, die sich der Idee nach peu à peu an die Oberfläche der Klassengesellschaft zurücktastet. Leider gelang es ihm nicht mehr, diese Bewegung zu vollenden; die klassentheoretische Debatte der kritischen Theorie ist wesentlich von diesem Erbe zwischen abstrakter-ökonomischer und konkreter-politischer Klassenanalyse bestimmt (vgl. Vester 2012). Marx und Engels ließen also weitgehend offen, wie aus der „Klasse an sich“ eine „Klasse für sich selbst" wird, klar ist lediglich, dass zur Erklärung einfache Ableitungen aus dem (Re-)Produktionsprozess des Kapitals nicht ausreichen.

In der weiteren Geschichte der marxistischen Theoriebildung überwog zunächst eine strukturelle Lesart des Klassenbegriffs. Diese war sowohl bei sozialdemokratischen als auch unter kommunistischen Theoretiker_innen der Arbeiterbewegung gängig. Die führenden Köpfe der Zweiten Internationale wie Karl Kautsky gingen davon aus, dass eine zunehmende Vereinheitlichung der Arbeiterklasse deren Handlungsfähigkeit verbessere - was der tatsächlichen Heterogenität der Lohnabhängigen zwar nicht gerecht wurde, aber die Klasse doch immerhin als selbstverständlichen Adressat politischer Bemühungen setzte (vgl. den Beitrag von Dorothea Schmidt in diesem Heft). Auch trage die Produktivkraftentwicklung dazu bei, dass mit den Großunternehmen bereits die materielle Grundlage für eine sozialistische Vergesellschaftung gegeben sei.

Noch mechanischer waren die Klassentheorien im Staatssozialismus: Die offizielle Staatsdoktrin der Sowjetunion ging lange Zeit davon aus, dass eine historisch-teleologische Stufenabfolge existiere. Den Produktionsverhältnissen der Urgemeinschaft folge die Sklaverei, dann der Feudalismus und schließlich der Kapitalismus; ähnlich wie in früheren Transformationen reife im Schoße der kapitalistischen Produktionsweise ein Widerspruch zwischen Produktionsverhältnissen und Produktivkraftentwicklung heran. Schließlich erstreite das revolutionäre Subjekt der Arbeiterklasse den Sozialismus. Der Zukunftsoptimismus beider Spielarten der Klassentheorie erwies sich durch den $\mathrm{Fa}$ schismus als verhängnisvolle Täuschung. Im wohlfahrtsstaatlich eingehegten Kapitalismus der Nachkriegsperiode wurde es dann in Westeuropa zeitweilig zum Konsens, 
dass die „nivellierte Mittelstandsgesellschaft" keine Klassen mehr kenne bzw. der Klassenkonflikt weitgehend aufgehoben worden sei; eine Erkenntnis, die nur wenige Theoretiker_innen wie Theodor W. Adorno bestritten (vgl. hierzu den Überblicksartikel von Hans-Günter Thien in diesem Heft).

Erst in den späten 1960er und 1970er Jahren kam es zu einer weiteren klassentheoretischen Diskussion. Sie verortete sich historisch in der Wellevon Klassenkämpfen dieser Periode und intervenierte in die Strategiedebatte der westeuropäischen kommunistischen und sozialistischen Parteien. Auch die Transformation des fordistischen Kapitalismus war ein Thema, wie es die damals weit verbreitete These von der Herausbildung einer professional-managerial class und die Theorie des staatsmonopolitischen Kapitalismus veranschaulichten. In Frankreich entwickelte sich im Umfeld von Louis Althusser und in kritischer Distanz zur Linie der Kommunistischen Partei Frankreichs eine angeregte Diskussion. Althussers Lektüre von Marx trug dazu bei, die Klassen als Ergebnis von klassenkampfvermittelten Prozessen der Reproduktion zu begreifen. Besondere Bedeutung kam dabei der Überlegung zu, dass die Individuen durch ideologische Staatsapparate als Subjekte angerufen und gleichzeitig in einer Weise unterworfen werden, dass sie die Produktionsverhältnisse tragen. Nicos Poulantzas schloss in seinen Arbeiten zu einer marxistischen Staatstheorie an Althussers Überlegungen an. Aus dieser Sicht gab es keine Klassen logisch und zeitlich „vor" dem Staat. Klassen sind immer auch politisch konstituiert. Kräfteverhältnisse zwischen Klassen verdichten sich derart in den Staatsapparaten, dass diese auch jeweils daran mitwirken, in welcher Weise sich Klassen bilden und Klassenkämpfe überhaupt in Begriffen der Klasse ausgetragen wird.
Es ist eine der unglücklichen Konstellationen der 1970er Jahre, dass E.P. Thompson in seiner Auseinandersetzung mit den Althusserianern im Umfeld der New Left Review und ihrer strukturalistischen Konzeption von Gesellschaft solche Affinitäten $\mathrm{zu}$ seinen eigenen Arbeiten verkannte. Er wollte Klasse weder als Kategorie noch als Struktur begriffen wissen, sondern betonte stattdessen, dass Klasse sich ,in der Tat so [definiert], wie sie in Erscheinung tritt" (1980: 268). Nicht Klassen seien der Ausgangspunkt, sondern ihre aktive (Selbst-)Herstellung, das re-making of class, im Klassenkampf sei „der universellere Begriff“" (ebd.: 267). Diese konzeptionell offene Herangehensweise ermöglichte auch die produktive Einbindung außerökonomischer Faktoren in die Klassenanalyse. Als besonders wirkungsmächtig ist hier das Konzept der „moralischen Ökonomi““ zu nennen, das Aufruhr und Klassenmobilisierung nicht aus objektiver Verelendung, sondern einer systematischen Verletzung von Legitimitätsvorstellungen erklärt.

Ein weiterer Ansatz kam aus der Diskussion um den Eurokommunismus, in der die Arbeiten von Antonio Gramsci rezipiert wurden. Ähnlich wie Thompson hatte der frühere PCI-Vorsitzende in seinen Gefängnisheften nicht die im engeren Sinne ökonomischen Faktoren der Klassenformierung betont. Seine Arbeiten widmen sich vielmehr der Frage, wie Staatlichkeit analysiert und auf Konsens beruhende Herrschaft gedacht werden kann und münden in der berühmten Formel: „Staat = politische Gesellschaft + Zivilgesellschaft, das heißt Hegemonie, gepanzert mit Zwang" (Gramsci 1992: 783). Auf die Klassenanalyse gewendet, bedeutet dies, dass es herrschenden wie subalternen Klassenfraktionen darum gehen muss, verallgemeinerbare Positionen zu vertreten, hinter denen sich ,historische Blöcke" als Kollektivsubjekte sammeln 
können. Diese Blöcke beruhen auf einer materiellen Grundlage und haben durchaus eine Klassenbasis - gehen aber nicht in ihr auf, sondern umfassen auch die kulturelle Hegemonieproduktion.

In den 1980er Jahren folgte eine erneute Verschiebung in der Diskussion. Angesichts der technologischen Veränderungen und der Entstehung von struktureller Arbeitslosigkeit konnte André Gorz von einer "Nicht-Klasse der Nicht-Arbeiter" sprechen. Das Proletariat wurde verabschiedet; im Anschluss an Ulrich Beck fand in den Sozialwissenschaften verbreitet die These von der Individualisierung Resonanz. Vielfach wurde die Aufmerksamkeit auch gefesselt von den neuen sozialen Bewegungen, deren Ziele (Umwelt, Geschlecht, Frieden) nicht ohne weiteres durch die soziale Herkunft der Aktiven erklärt werden konnten. Deswegen wurden die Konflikte im Anschluss an die Arbeiten von Ronald Inglehart missverständlicher Weise als „postmaterialistisch “ bezeichnet. Am Beispiel der Sorgearbeit beschreiben Brigitte Aulenbacher u.a. in diesem Heft wie nicht nur klassen-, sondern auch geschlechts- und ethnizitätsbasierte Herrschaftsformen ineinander greifen und ihrerseits Sorgeregime konstituieren.

Im Lager der kritischen Theorie wurden v.a. Ernesto Laclau und Chantal Mouffe (1991) mit ihrem diskurstheoretisch informierten Unternehmen, das „letzte Bollwerk des Essentialismus: die Ökonomie“ (ebd.: 112) anzugreifen, einflussreich. Klassen werden in diesem Fall als erfolgreiche Anrufungen, als das kontingente Ergebnis von langen Artikulationsketten gedacht, die sich unter gewissen Umständen formieren und auch wieder verschwinden können. Zwar konnte auf diese Weise der Klassenreduktionismus mancher früherer Klassentheorie problematisiert werden. Doch erweist sich der Ansatz letztlich als begriffslos gegenüber gesellschaftlichen Strukturen, z.B. der Produktionsweise, die bei Gramsci - dem Stichwortgeber der für Laclau/Mouffe zentralen Hegemoniefrage - noch im Mittelpunkt stand. Im Folgeheft PROKLA 176 argumentiert Christian Lotz, dass die „harte“, außerdiskursive Gewaltförmigkeit liberaler Klassengesellschaften in der postmarxistischen Perspektive aus dem Blick gerät.

Erst aus dem zeitlichen Abstand wird Pierre Bourdieus Beitrag zur Diskussion der 1980er Jahre deutlich: während (nicht nur) die deutsche Soziologie von Klassen kaum noch reden wollte, wo sie die Verschiedenheit und Dynamik von Konsum- und Lebensstilen ausmalte und von Erlebnis- und Multioptionengesellschaft redete, erkannte er fortbestehende Strukturen der Klassengesellschaft. Die sozialen Unterschiede, die sich aus der Ausstattung der Akteure mit ökonomischen und kulturellen Ressourcen zunächst „auf dem Papier" (Bourdieu 1985: 12) ergeben, werden von ihnen zu Unterscheidungen (in Fragen des Essens, des Wohnens, des Kunstgeschmacks etc.) gemacht und bilden qua Klassifikations-, Wahrnehmungs- und Bedeutungspraxen der Akteure Klassen. Die Analyse der Ökonomie wird bei Bourdieu derart ergänzt durch die der „kulturellen Produktionsverhältnisse" (1985: 31) und der symbolischen Herrschaft. Mit dieser, auch stark an Weber orientierten Vorgehensweise, kann Bourdieu zwar die Eindimensionalität mancher marxistischer Klassentheorie überwinden, er beschreibt allerdings weniger „die französische Klassengesellschaft (im präzisen Sinne des Wortes), [als] vielmehr deren ständische Ausformung" (Rehbein 2004: 26). Unabhängig von dieser Kritik könne mit dem Bourdieu'schen Instrumentarium, so argumentiert unser Autor Christian Baron, auch der Begriff des Klassismus vom Kopf auf die Füße gestellt werden. 
Lenkten die Arbeiten von Bourdieu das Augenmerk auf die kulturellen Repräsentationen der Klassengesellschaft, so bemühte sich ungefähr zur selben Zeit E.O. Wright eine andere Leerstelle der Klassentheorie ausfüllen: die „peinliche Existenz' der Mittelklasse“ (1985: 35) sollte durch ein universalisiertes Ausbeutungskonzept erklärt werden. Dafür führte Wright neben dem (Spezial-)Fall der Ausbeutung qua Mehrwertabschöpfung noch drei weitere Dimensionen ein: ungleiche Verfügung über Arbeitskraft, Organisationskompetenz und Qualifikation. Demnach bilden sich mittlere Klassenlagen dann heraus, wenn sich verschiedene Ausbeutungsdimensionen überlappen und einzelne Gruppen ,in Bezug auf eine bestimmte Dimension der Ausbeutungsverhältnisse zu den Ausgebeuteten zählen, während sie in Bezug auf eine andere Dimension ausgebeutet werden“ (ebd.: 47). John Roemer (1981), ein weiterer Vertreter des analytischen Marxismus, versuchte den Ausbeutungsbegriff nicht werttheoretisch zu fassen, sondern mit Hilfe der Logik und analytischer Philosophie zu entwickeln. Im deutschsprachigen Diskurs wurde auf die Begriffe der Dienstoder Versorgungsklassen zurückgegriffen, um die (mittel-)klassenbildenden Effekte des Wohlfahrtstaats und neue, kollektive geteilte Lebensführungsmuster zu erklären (vgl. z.B. Vogel 2009: 116ff.). ${ }^{1}$

Zusammengefasst: Die Klassentheorie wurde nach einer angeregten Diskussion in den 1960er und 1970er Jahren im Folgejahrzehnt nur noch vereinzelt weiter ausgearbeitet. Wenn sie nicht de facto aufgegeben wurde (Laclau/Mouffe), wurde

1 Leider vermissen wir im vorliegenden Heft einen Beitrag, der auf die aktuelle Transformation der Mittelklassen abhebt, die sich u.a. einem zunehmenden Prekarisierungsdruck ausgesetzt sieht. nun meist aufkulturell-lebensweltliche Aspekte fokussiert (Bourdieu); strukturellökonomische Lesarten (Wright) waren kaum noch präsent.

\section{Die aktuelle Diskussion}

Nach einer langen Phase des Niedergangs der Klassentheorie gibt es heute wieder aktuelle Arbeiten (für einen aktuellen Überblick vgl. z.B. Thien 2011 oder das aktuelle Socialist Register 2014). Diese versuchen meist, die derzeitigen Entwicklungstendenzen des Kapitalismus zu erklären und greifen hierfür auf klassentheoretische Bezüge zurück. Erneut wurden eher strukturalistisch-ökonomisch und praxeologisch-politische Ansätze ausgearbeitet. Ein Diskussionsstrang widmet sich der Transnationalisierung von Klassenstrukturen. Verschiedene neo-gramscianische Autoren wie William Robinson oder Bastiaan van Apeldoorn gehen davon aus, dass durch den Globalisierungsschub der letzten Jahrzehnte grenzüberschreitende Akkumulationsprozesse die nationalen Wirtschaftskreisläufe als Referenzrahmen abgelöst haben (z.B. Robinson 2012, Apeldoorn 2013). Große transnationale Konzerne dominierten heute die Weltwirtschaft. Eine neue transnationale Kapitalistenklasse betreibe in internationalen Foren und Organisationen wie der EU ein aktives Agendasetting. Sie habe über Elitenetzwerke wie das World Economic Forum ihre Interessen verallgemeinert und kontrolliere die Regulierung der Weltmärkte. Auch in der Bearbeitung der Krise seit 2008/9 spielte diese Klasse eine zentrale Rolle, wie Mathis Heinrich in seinem Beitrag am Beispiel der EU herausarbeitet. Die Diskussion um die transnationale Kapitalistenklasse bezieht sich auf einen strukturalistisch-funktionalistischen Klassenbegriff. Bisher blieb aber weitgehend offen - wie Michael Hartmann (2008) kritisch anmerkte -, ob sich die 
transnationalen Vermögensbesitzer und -verwalter tatsächlich als eine gemeinsame Klasse wahrnehmen; empirische Daten weisen z.B. weiterhin auf überwiegend national strukturierte Karrierewege und Lebenswelten hin.

Doch nicht nur zu den herrschenden, auch zu den Unterklassen ist eine neue Diskussion entstanden. Dabei dominieren vor allem Arbeiten zur Prekarisierung der subalternen Klassen, die die subjektive Verarbeitung von unsicheren Arbeits- und Lebensverhältnissen untersuchen (z.B. Castel/Dörre 2009). Prekarisierung ist in diesen Studien ein relationaler Begriff, die Prekaritätserfahrung ist meist mit dem Leitbild eines stabilen Normalarbeitsverhältnisses verbunden. Klassentheoretisch wurde dabei die Frage aufgeworfen, ob ein neues „Prekariat“ im Entstehen begriffen sei (Standing 2011). Bei dieser Begriffspolitik ist allerdings eine gewisse Neigung zu erkennen, äußerst unterschiedliche soziale Gruppen zusammenzubringen. Doch die gleiche prekäre Lage von „Putzmob“ und Laptop ergibt sich nicht von selbst. Andererseits trifft der Begriff das Selbstverständnis vieler Betroffener; es könnte also ein Prozess eines (bewegungspolitischen) class-making einsetzen, der aber materialistisch untersetzt bleiben muss, wie Peter Bescherer in seinem Beitrag diskutiert. Denn Prekarität ist keineswegs „überall“ (Bourdieu), das Prekaritätsrisiko ist in unteren sozialen Lagen weitaus größer und wird zudem politisch hergestellt (Dörre u.a. 2013), sodass eine Tendenz zur Verfestigung des prekären Sektors an den Rändern der Gesellschaft existiert. Ob jedoch eine Unterklasse als gesellschaftlicher Akteur auftritt, bleibt fraglich.

Zuletzt sind - gerade nach der Finanz- und Wirtschaftskrise 2008 - auch die zunehmenden sozialen Proteste zu einem Thema der Klassentheorie geworden. Ein wichtiger Bezugspunkt in dieser Debatte spielte die historische Studie von Beverly Silver (2005) zur weltweiten Arbeiterunruhe. Sie wies nach, dass sich das Gros der Klassenkämpfe in die aufstrebenden Schwellenländer - insbesondere nach China als Epizentrum der weltweiten Arbeiterunruhe (vgl. PROKLA 161) - verlagert hat und vermutete, dass eine neue weltweite Konfliktwelle drohe. Die Protestwelle nach der Weltwirtschaftskrise scheint Silvers These zu bestätigen; allerdings sind die Protestierenden nicht nur Arbeiter_innen, sondern stammen auch aus anderen sozialen Gruppen, zudem sind Weltregionen wie der Nahe Osten oder Südeuropa betroffen, die Silver selbst nicht ins Zentrum der Analyse gerückt hatte. Insgesamt deutet die neue Protestwelle darauf hin, dass der Klassenkonflikt - zumindest in Westeuropa - aufsplittert. Es ist vermehrt zu nicht-normierten Konflikten gekommen, die aus den bewährten institutionellen Bahnen ausbrechen: Massenproteste und politische Streiks, Riots und „wilde“ betriebliche Konflikte haben sich in den vergangenen Jahren gehäuft (Schmalz/Liebig i.E.). Dieser Zersplitterung der sozialen Konflikte geht Steffen Liebig in seinem Artikel am Beispiel der britischen Riots 2011 auf die Spur.

Für die Redaktion: PeterBescherer, Steffen Liebig, Stefan Schmalz

\section{Literatur}

Apeldoorn, Bastiaan van (2013): The European Capitalist Class and the Crisis of its Hegemonic Project, In: Socialist Register 2014, Jg. 50, S. 189-206.

Beck, Ulrich; Poferl, Angelika (Hrsg.) (2010): Große Armut, großer Reichtum, Frankfurt. Bollmann, Ralph; Kloepfer, Inge (02.06.2013): Die neue Klassengesellschaft. In: http:// www.faz.net/aktuell/wirtschaft/menschenwirtschaft/chancengerechtigkeit-die-neueklassengesellschaft-12204524.html 
Bourdieu, Pierre (1985): Sozialer Raum und 'Klassen'. Leçon sur la leçon, Frankfurt/M.

Demirović, Alex (2008): Die Konflikttheorie von Karl Marx. In: Bonacker, Thorsten (Hg.): Sozialwissenschaftliche Konflikttheorien. Wiesbaden, S. 47-64.

Dörre, Klaus (2010): Soziale Klassen im Prozess kapitalistischer Landnahmen. In: Bude, Heinz; Damitz, Ralf M.; Koch, André (Hrsg.): Marx. Ein toter Hund? Gesellschaftstheorie reloaded. Hamburg, S. 198-236.

Castel, Robert; Dörre, Klaus (2009): Prekarität, Abstieg, Ausgrenzung: Die soziale Frage am Beginn des 21. Jahrhunderts. Frankfurt/M.

Dörre, Klaus u.a. (2013): Bewährungsproben für die Unterschicht? Soziale Folgen aktivierender Arbeitsmarktpolitik. Frankfurt/M.

Gallie, Walter Bryce (1956): Essentially Contested Concepts. In: Proceedings of the Aristotelian Society, Nr. 56, S. 167-198.

Giddens, Anthony (1984): Die Klassenstruktur fortgeschrittener Gesellschaften. Frankfurt/M.

Gramsci, Antonio (1992): Gefängnishefte Bd. 4. Hamburg.

Hartmann, Michael (2008): Transnationale Klassenbildung, in: Berger, Peter A.; Weiss, Anja (Hg.): Transnationalisierung sozialer Ungleichheit, Wiesbaden, S. 241-258.

Heinrich, Michael (1994): PROKLA - oder wie ist heute eine linke, wissenschaftliche Zeitschrift möglich? In: Thien, Hans-Günter (Hg.): Bücher, nichts als Bücher. Münster, S. 104-110.

Koch, Max (1994): Vom Strukturwandel einer Klassengesellschaft : theoretische Diskussion und empirische Analyse. Münster.

Kohlmorgen, Lars (2004): Regulation, Klasse, Geschlecht: die Konstituierung der Sozialstruktur im Fordismus und Postfordismus. Münster.

Laclau, Ernesto; Mouffe, Chantal (1991): Hegemonie und radikale Demokratie: Zur Dekonstruktion des Marxismus. Wien.

Offe, Claus (1972): Strukturprobleme des kapitalistischen Staates. Frankfurt/M.

NYT (26.11.2006): In Class Warfare, Guess Which Class Is Winning. In: http:// www.nytimes.com/2006/11/26/business/ yourmoney/26every.html?ex $=1165554000$ \&en $=02$ ed 48 ael 473 efe0\&ei $=5070 \& \_r=0$.
Piketty, Thomas (2014): Capital in the TwentyFirst Century. Harvard.

PROKLA Redaktion (1985): Editorial. In: PROKLA, Nr. 58, Jg. 15, H. 1, S. 1-4.

Karl-Siegbert Rehberg (2004): Die unsichtbare Klassengesellschaft. Eröffnungsvortrag zum 32. Kongress der Deutschen Gesellschaft für Soziologie. In: Ders. (Hg.): Soziale Ungleichheit, kulturelle Unterschiede. Verhandlungen des 32. Kongresses der Deutschen Gesellschaft für Soziologie in München 2004. Frankfurt/M, S. 19-38.

Robinson, William (2012): Global Capitalism Theory and the Emergence of Transnational Elites. In: Critical Sociology Jg. 38, H. 3, S. 349-363.

Roemer, John (1981): Analytical foundations of Marxian economic theory. Cambridge.

Schmalz, Stefan; Liebig, Steffen (i.E): Ein neuer Protestzyklus? Zum Wandel des sozialen Konflikts in Westeuropa. In: Dörre, Klaus et al. (Hrsg.): Arbeit in Europa. Marktfundamentalismus als Zerreißprobe. Frankfurt/M.

Silver, Beverly J. (2005): Forces of Labor. Arbeiterbewegungen und Globalisierung seit 1870. Berlin.

Standing, Guy (2011): The Precariat. The New Dangerous Class. London.

Thien, Hans-Günter (Hrsg.) (2011): Klassen im Postfordismus. Münster.

Thompson, Edward P. (1980): Plebeische Kultur und moralische Ökonomie Aufsätze zur englischen Sozialgeschichte des 18. und 19. Jahrhunderts. Frankfurt/M.

Vester, Michael (2008): Klasse an sich/für sich. In: Haug, Frigga; Haug, Wolfgang Fritz; Jehle, Peter (Hg.): Historisch-Kritisches Wörterbuch des Marxismus. Band 7/1. Kaderpartei bis Klonen. Hamburg, S. 736-775.

Vogel, Berthold (2009): Wohlstandskonflikte. Soziale Fragen, die aus der Mitte kommen. Hamburg.

Wehler, Hans-Ulrich (07.02.2013): Wachsende Ungleichheit. In: http://www.zeit. de/2013/07/Essay-Bundesregierung-Armuts-und-Reichtumsbericht.

Wright, Erik Olin (1985): Wo liegt die Mitte der Mittelklasse? In: PROKLA, Nr. 58, Jg. 15, H. 1, S. 35-62. 